\title{
Incidence of Proximal Adjacent Failure in Adult Lumbar Deformity Correction Based on Proximal Fusion Level
}

\author{
Jin-Hyok Kim, Sung-Soo Kim, Se-II Suk \\ Seoul Spine Institute, Inje University Sanggye Paik Hospital, Seoul, Korea
}

\begin{abstract}
Study Design: Retrospective study.
Purpose: To review the results and proximal adjacent problems of long fusion (more than 4 levels) according to the level of proximal fusion (L2 T9) in adult lumbar deformity using pedicle screw fixation.

Overview of Literature: There are few written reports concerning proximal adjacent segmental failure according to the level of proximal fusion in adult lumbar deformity.
\end{abstract}

Methods: The radiographs and clinical records of thirty-five patients ( 30 females, 5 males) of adult lumbar deformity with more than 2-year follow-up after surgery were analyzed. The average age was 62 years (range, 38 75). All patients were divided into three groups according to the level of proximal fusion: Group 1 ( $n=14)$ fusion up to L1 or L2; Group 2 ( $n=14)$ fusion up to T11 or T12; and Group 3 ( $n=7$ ) fusion up to T9 or T10.

Results: The preoperative coronal curve of $28 \pm 14^{\circ}$ was corrected to $9 \pm 7^{\circ}$ immediately after surgery and $11 \pm 7^{\circ}$ at the final follow-up. The preoperative local kyphosis of $24 \pm 12^{\circ}$ was corrected to $-1 \pm 10^{\circ}$ immediately after surgery and $1 \pm 11^{\circ}$ at the final follow-up. The lumbar lordosis was $14 \pm 18^{\circ}$ before surgery; $27 \pm 11^{\circ}$ after surgery; and $16 \pm 12^{\circ}$ at the final follow-up. The parameters of coronal and sagittal balance were improved in all patients after surgery, except one patient in group 2 who showed coronal imbalance due to over-correction. Sagittal imbalance at the most recent follow-up was detected in 10 patients with significant difference between the groups; 5 (36\%) in Group 1, 5 (36\%) in Group 2, and none in Group 3. Proximal adjacent segmental problems that are consisted with proximal disc degeneration with kyphosis, compression fractures above the fusion and screw failure proximal to the end of the fusion were observed in 15 patients with significant difference between the groups; $7(50 \%)$ in Group $1,7(50 \%)$ in Group 2, and 1 (14\%) in Group 3. There was 1 superficial infection and 2 transient neurologies.

Conclusions: Fusion up to throacolumbar junction (L2 T11) in surgical treatment of adult lumbar deformity had more proximal adjacent problems with poorer results. Fusion higher than T10 is recommended for adult lumbar deformity.

Key Words: Adult lumbar deformity, Proximal fusion level, Proximal adjacent problem, Adjacent segmental failure

\section{Introduction}

With the increasing geriatric population, adult lumbar deformity has become a common and occasionally disabling condition of the spine. Advanced degenerative disease in the facet joints and degenerative disc disease can lead to pain, spinal stenosis, and deformity ${ }^{1-3}$. The initial treatment of patients with adult lumbar deformity is nonoperative ${ }^{4}$. However, if conservative measures fail, surgical treatment would be considered. Surgical procedures should be designed to provide maximum stability with amelioration of neurology, often needing long fusions up to thoracolumbar junction.

Corresponding author: Jin-Hyok Kim, MD

Seoul Spine Institute, Inje University Sanggye Paik Hospital

761-1 Sanggye-dong, Nowon-gu, Seoul, 139-707, Korea

Tel: +82-2-950-1288, Fax: +82-2-3392-1101, E-mail: kjh1026@ sanggyepaik.ac.kr

This work was supported by the 2006 Inje University research grant. 
Fusion for adult lumbar deformity has been shown to provide a significantly greater decrease in pain and fatigue for patients treated surgically compared to those with adult lumbar deformity who were not treated ${ }^{5}$. Compared with adolescent deformity, fusion for adult deformity is often associated with high rates of complications, including pseudarthorosis, instrumentation failure, junctional problem, and higher morbidity ${ }^{6.8}$. The major concerns in long fusions for adult lumbar deformity have focused on the distal fusion level and distal instrumentation failure ${ }^{9-11}$, but there are few written reports concerning proximal adjacent segmental failure according to the level of proximal fusion in adult lumbar deformity.

It is the purpose of this study to review the results of long posterior fusion with pedicle screw fixation, according to the level of proximal fusion extent in adult lumbar deformity, and to make suggestions regarding the future management of adult lumbar deformities.

\section{Materials and Methods}

Thirty-five adult lumbar deformity patients were retrospectively reviewed after a minimum follow-up of 2 years (range, 2 5.8 years). The apex of the deformity was below L1 on coronal and/or sagittal planes. All the patients were subjected to posterior fusion with pedicle screw fixation. The proximal fusion end was the thoracolumbar junction from T9 to L2, and the distal end was L5 or S1. Patients who had distal instrumentation failure and distal adjacent segmental problems were excluded. There were 5 males and
30 females. The average age at the operation was 62 years (range: 38 75 years). Of the 35 patients, 20 had degenerative lumbar scoliosis, 10 had degenerative lumbar kyphosis, and 5 had postoperative flat back. They were divided into three groups according to the level of proximal fusion: Group 1 (n=14) with fusion up to L1 or L2; Group 2 ( $n=14)$ with fusion up to T11 or T12; and Group $3(\mathrm{n}=7)$ with fusion up to T9 or T10. Demographics and etiologic diagnosis in three groups were shown in Table 1.

The clinical records were reviewed for operation time, average blood loss, functional improvement, and complications. Radiographic measures were made from 36-inch standing anteroposterior and lateral radiographs taken before surgery, within 1 month after surgery and at the most recent follow-up to assess deformity correction, spinal balance, complications related to the instrumentation, and any evidence of the proximal junctional problem. When the patient had a reoperation for proximal adjacent problem, radiographic evaluation at the most recent follow-up was based on radiographs taken before reoperation. Coronal curve was measured by the Cobb method. Coronal balance was measured by the distance between the $\mathrm{C} 7$ plumb line and the center sacral line and coronal imbalance was defined as this parameter $\geq 30 \mathrm{~mm}$. Local kyphosis was measured by the Cobb method in the sagittal plane. Lumbar lordosis was measured by the Cobb method from the superior end plate of T12 to the upper end plate of S1. Sagittal balance was measured by the distance between the $\mathrm{C} 7$ plumb line and the posterior superior corner of $\mathrm{S} 1$ and sagittal imbalance was defined as this parameter $\geq 50 \mathrm{~mm}$. Patients who had preoperative coronal curve of more than

Table 1. Demographics and etiologic diagnosis

\begin{tabular}{lccc}
\hline & Group 1 (n=14) & Group 2 (n=14) & Group 3 (n=7) \\
\hline Age (years) & $63(39 \sim 74)$ & $60(38 \sim 71)$ & $66(59 \sim 75)$ \\
Female / Male & $12 / 2$ & $12 / 2$ & $6 / 1$ \\
Follow-up (months) & $47(24 \sim 70)$ & $50(26 \sim 70)$ & $45(24 \sim 69)$ \\
Diagnosis & & & 4 \\
DLS & 8 & 5 & 2 \\
DLK & 4 & 2 & 1 \\
Postop. flatback & 2 & 5 & 4
\end{tabular}

DLS: degenerative lumbar scoliosis, DLK: degenerative lumbar kyphosis, Postop. flatback: postoperative flatback.

Table 2. The number of patients with scoliosis or kyphosis according to the group

\begin{tabular}{lcccc}
\hline & Group 1 & Group 2 & Group 3 & Total \\
\hline Scoliosis & 9 & 7 & 4 & 20 \\
Kyphosis & 7 & 12 & 4 & 23 \\
\hline
\end{tabular}


$15^{\circ}$ were defined as scoliosis, and local kyphosis of more than $10^{\circ}$ measured from consecutive 3 segments or more of vertebra as kyphosis (Table 2). Eight patients had kyphoscoliosis (coronal curve more than $15^{\circ}$ and local kyphosis more than $10^{\circ}$ ). Proximal adjacent segmental problems included adjacent segment degeneration, compression fractures adjacent to the fusion mass, or proximal screw failure. Adjacent segment degeneration was defined by the presence of at least two of the following radiographic conditions during postoperative follow-up; progressive disc height narrowing of more than $2 \mathrm{~mm}$, decrease in lordosis or increase in kyphosis of more than $5^{\circ}$, spur formation and sclerosis of the adjacent end plate, or translation of more than $2 \mathrm{~mm}$. Clinical outcomes were assessed with the Oswestry score.

Surgical procedures were performed by the senior author through a posterior route, including decompression of the stenotic levels and correction and stabilization of the spine with segmental pedicle screw fixation. Decompression was carried out in 27 patients. Correction and stabilization without decompression was carried out in 8 patients. Realignment osteotomies were required in 8 patients to correct rigid curves.

The proximal fusion level was decided upon the preoperative curve angle and characteristics. The basic principle of instrumentation in degenerative lumbar deformity was to avoid ending the instrumentation at an area of junctional kyphosis or at the level of retrolisthesis or spondylolisthesis.

\section{Results}

There were no significant differences in demographics nor the magnitude of deformity and preoperative truncal balance between the 3 groups. As a whole, the mean operation time was $201 \pm 44$ minutes with a blood loss of $2971 \pm$ $1773 \mathrm{~mL}$. In Group 1, the mean operation time was $190 \pm$ 50 minutes with a blood loss of $2909 \pm 999 \mathrm{~mL}$. In Group 2 , the mean operation time was $198 \pm 26$ minutes with a blood loss of $2717 \pm 1715 \mathrm{~mL}$, and $216 \pm 49$ minutes with a blood loss of $3407 \pm 2324 \mathrm{~mL}$ in Group 3 .

\section{Deformity correction}

The number of patients with scoliosis or kyphosis in each group was shown in Table 2. In those with scoliosis $(\mathrm{n}=20)$, the preoperative coronal curve of $28 \pm 14^{\circ}$ was corrected to $9 \pm 7^{\circ}$ at immediate postoperative assessment, and $11 \pm 7^{\circ}$ at the most recent follow-up. The changes of scoliosis before and after surgery based on groups were described in Table 3. The loss of scoliosis correction during the followup in Group 1 was greater than others, even if the preoperative Cobb angle of Group 1 is the least. In those patients with kyphosis $(n=23)$, the preoperative local kyphosis of 24 $\pm 12^{\circ}$ was corrected to $-1 \pm 10^{\circ}$ at immediate postoperative assessment, and $1 \pm 11^{\circ}$ at the most recent follow-up. The

Table 3. Coronal curve magnitude of scoliosis patients

\begin{tabular}{lccc}
\hline & Group $1(\mathrm{n}=9)$ & Group 2 $(\mathrm{n}=7)$ & Group 3 $(\mathrm{n}=4)$ \\
\hline Preoperative Cobb angle $\left({ }^{\circ}\right)$ & $20.7 \pm 3.8$ & $37.0 \pm 19.6$ & $30.8 \pm 11.5$ \\
IMPO Cobb angle $\left(^{\circ}\right)$ & $4.8 \pm 4.4$ & $13.5 \pm 7.8$ & $11.0 \pm 1.4$ \\
Correction rate $(\%)$ & $77.4 \pm 17.5$ & $61.2 \pm 22.7$ & $59.6 \pm 18.3$ \\
Final Cobb angle $\left({ }^{\circ}\right)$ & $7.0 \pm 5.8$ & $14.6 \pm 7.0$ & $12.0 \pm 3.7$ \\
Correction rate $(\%)$ & $66.0 \pm 26.1$ & $57.2 \pm 21.6$ & $55.7 \pm 25.0$ \\
LOC $(\%)$ & 11.3 & 6.4 & 3.9 \\
\hline
\end{tabular}

IMPO: immediate postoperative, LOC: loss of curve correction during the follow-up.

Table 4. Sagittal curve magnitude of kyphosis patients

\begin{tabular}{lccc}
\hline & Group 1 $(\mathrm{n}=7)$ & Group 2 $(\mathrm{n}=12)$ & Group 3 $(\mathrm{n}=4)$ \\
\hline Preoperative local kyphosis $\left(^{\circ}\right)$ & $14.6 \pm 3.3$ & $28.4 \pm 11.3$ & $26.5 \pm 14.7$ \\
IMPO local kyphosis $\left({ }^{\circ}\right)$ & $-6.7 \pm 7.7$ & $2.7 \pm 9.9$ & $-3.8 \pm 10.3$ \\
Correction rate $\left({ }^{\circ}\right)$ & $21.3 \pm 8.1$ & $25.8 \pm 8.4$ & $30.3 \pm 12.4$ \\
Final local kyphosis $\left({ }^{\circ}\right)$ & $-2.3 \pm 7.7$ & $4.3 \pm 12.1$ & $-2.8 \pm 10.8$ \\
Correction rate $\left({ }^{\circ}\right)$ & $16.9 \pm 8.3$ & $24.2 \pm 13.1$ & $29.3 \pm 14.8$ \\
LOC $\left(^{\circ}\right)$ & 4.4 & 1.6 & 1.0 \\
\hline
\end{tabular}

IMPO: immediate postoperative, LOC: loss of curve correction during the follow-up, '-' (minus) means lordosis. 
changes of local kyphosis before and after surgery based on groups were described in Table 4. The loss of kyphosis correction during the follow-up in Group 1 was greater than others, even if the preoperative local kyphosis of Group 1 is the least. Lumbar lordosis was $14 \pm 18^{\circ}$ before surgery; 27 $\pm 11^{\circ}$ at immediate postoperative assessment, and $16 \pm 12^{\circ}$ at the most recent follow-up.

\section{Balance}

Both the coronal and sagittal balance were improved at immediate postoperative assessment in all patients except one in Group 2 who showed coronal imbalance due to overcorrection. Coronal balance was well maintained during follow-up in all groups. Sagittal imbalance was detected in 10 patients at the most recent follow-up with significant differ- ence between 3 groups ( $\mathrm{p}<0.05) ; 5$ out of 14 (36\%) in Group 1; 5 out of 14 (36\%) in Group 2; and none in Group 3.

\section{Proximal adjacent segmental problems}

Proximal adjacent segmental failures were observed in 15 patients during follow-up with significant difference between 3 group ( $\mathrm{p}<0.05)$; 7 out of $14(50 \%)$ in Group 1,7 out of $14(50 \%)$ in Group 2, and 1 out of 7 (14\%) in Group 3 (Table 5) (Figs. 1-3). Adjacent segment degeneration developed in 12 patients at the most recent follow-up; 5 in Group 1, 6 in Group 2, and 1 in Group 3. Progressive narrowing of disc height more than $2 \mathrm{~mm}$ was the most common type. Compression fracture adjacent to the fusion mass occurred in 2 patients; each in Group 1 and 2. Proximal screw failure was found in 1 patient in Group 1.

Table 5. The number of patients with proximal adjacent segmental failures

\begin{tabular}{lcccc}
\hline & Group 1 $(\mathrm{n}=14)$ & Group 2 $(\mathrm{n}=14)$ & Group 3 (n=7) & Total \\
\hline PASD & 5 & 6 & 1 & $12(34 \%)$ \\
Comp. Fx. & 1 & 1 & 0 & $2(6 \%)$ \\
Screw failure & 1 & 0 & 0 & $1(3 \%)$ \\
\hline Total & $7(50 \%)$ & $7(50 \%)$ & $1(14 \%)$ & $15(43 \%)$ \\
\hline
\end{tabular}

PASD: proximal adjacent segmental degeneration, Comp. Fx.: compression fracture above the fusion, Screw failure: screw failure at the proximal end of the fusion.
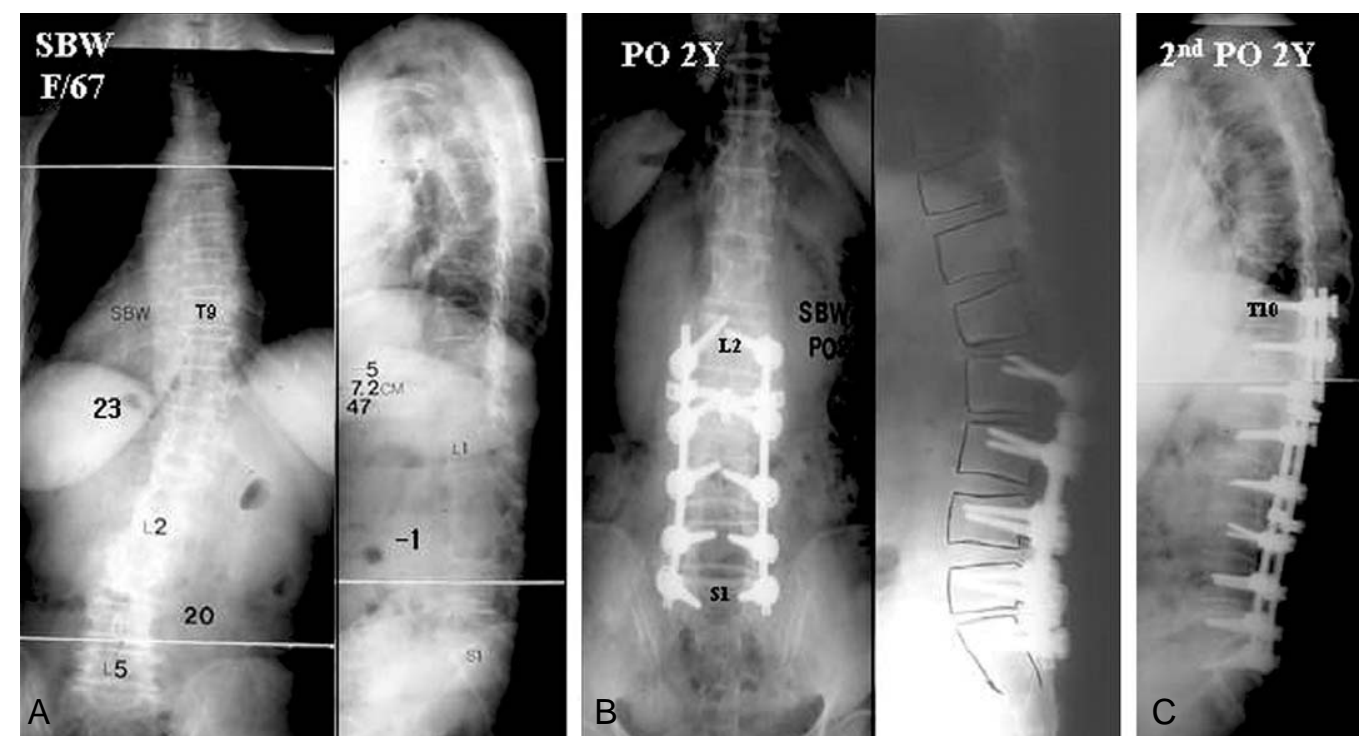

Fig. 1. A 67-year-old woman with low back pain, radicular leg pain and claudication. (A) The preoperative radiographs demonstrated a lumbar scoliosis of $20^{\circ}$ and lumbar lordosis of $1^{\circ}$. (B) She underwent posterior decompression L4 to S1, pedicle screw instrumentation and fusion to L2 to S1. The radiographs taken at 2 years after surgery showed proximal adjacent problem with junction kyphosis. (C) Extension of fusion to T10 with pedicle screw instrumentation was performed. In the radiograph taken at 2 year after reoperation, there was no proximal adjacent problem. 


\section{Other results and complications}

The preoperative Oswestry scores of $60.5 \pm 18.4$ in Group 1, 62.4 \pm 15.7 in Group 2 and 55.3 \pm 12.6 in Group 3 were improved to $45.6 \pm 20.7,48.2 \pm 16.4$ and $35.6 \pm 10.3$ at the most recent follow-up, respectively. There was no statistical significant difference in the change of Oswestry score among three groups $(\mathrm{p}>0.05)$.

Complications were encountered in 3 patients. One superficial infection resolved with drainage and antibiotic therapy without long-term sequelae. Two patients with cauda equina syndrome secondary to hematoma underwent an immediate surgical decompression and fully recovered within 6 months after surgery.
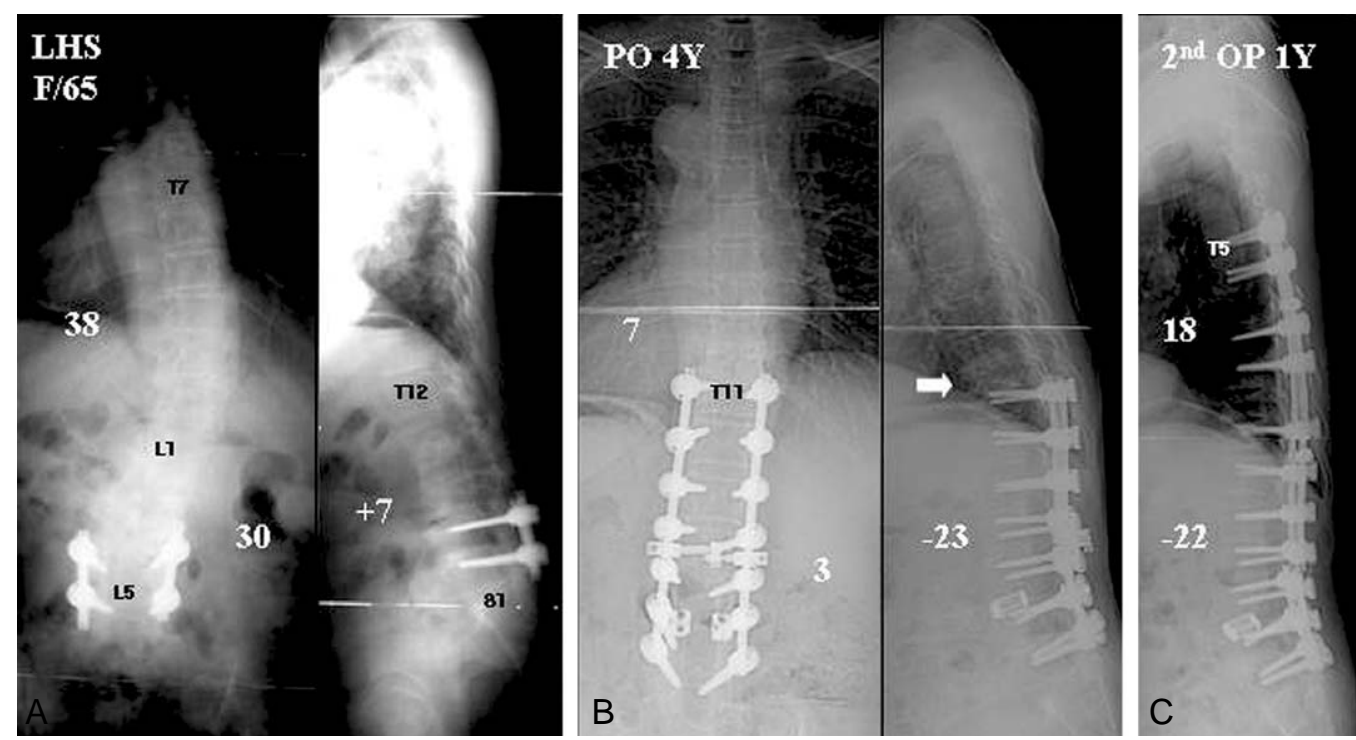

Fig. 2. A 65-year-old woman with worsening low back pain and claudication despite previous surgery of L4-5. (A) The preoperative radiographs demonstrated a lumbar scoliosis of $30^{\circ}$ and lumbar kyphosis of $7^{\circ}$. (B) She underwent posterior decompression L2 to L5, posterior lumbar interbody fusion at L3-4 with metal cage, pedicle screw instrumentation and fusion to T11 to S1 with correction of her deformity. The radiographs taken at 4 years after surgery showed proximal adjacent problem with degeneration and kyphosis. (C) Extension of fusion to T5 with pedicle screw instrumentation was performed. In the radiograph taken at 1 year after reoperation, there was no proximal adjacent problem.
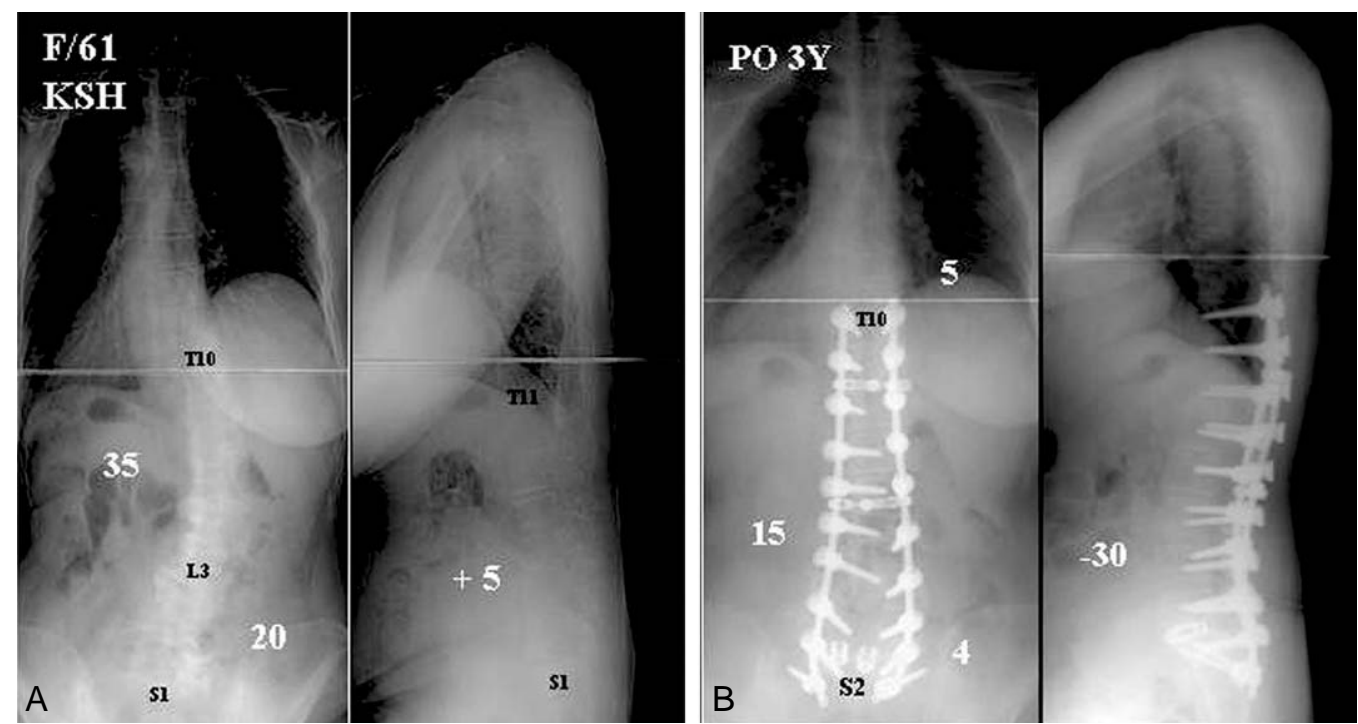

Fig. 3. A 61-year-old woman with low back pain, radicular leg pain and claudication. (A) The preoperative radiographs demonstrated a lumbar scoliosis of $20^{\circ}$ and lumbar kyphosis of $5^{\circ}$. (B) She underwent posterior decompression L4 to S1, posterior lumbar interbody fusion at L5-S1 with metal cage, pedicle screw instrumentation and fusion to T9 to S2 with correction of her deformity. In the radiograph taken at 3 year after operation, there was no proximal adjacent problem. 


\section{Discussion}

The prevalence of lumbar deformities had been reported to range from $2.5 \%$ to $15 \% \%^{3,12,13}$. In our experience ${ }^{14}$, the prevalence was $4.4 \%$ in adults and $8.6 \%$ in those over 60 years old, with a rapid increase after the sixth decade. Most of the adult lumbar deformities develop as a consequence of rapid asymmetrical degeneration of lumbar intervertebral discs and facet joint degeneration ${ }^{15,16}$. In contrast to the asymptomatic adolescent with scoliosis, patients with degenerative deformity often present with mechanical lower back pain, radicular pain and/or neurogenic claudication' As disc degeneration is more rapid in mobile segments, the apex is usually below L1 and the major deformity is in the lumbar region with short reciprocating curves above or below. Attempts should be made to treat patients with adult lumbar deformity non-operatively, but if conservative measures fail, surgical treatment should be considered. Surgical procedure should be designed to provide maximum stability with amelioration of neurology. All diseased segments should be stabilized if possible, and the spine should be balanced after surgery. As the surgical treatment of adult lumbar deformity often needs a long fusion up to the thoracolumbar junction, it is one of most morbid and challenging procedure for spine surgeons.

The results of surgical intervention in adult lumbar deformity have been varied, and only recently improved to an acceptable level. These improvements have resulted, in part, from a greater understanding of the need to balance the spine in both the coronal and sagittal planes of all diseased segments with fusion. Improvements in fixation have been important, beginning with Harrington instrumentation, evolving through Luque segmental instrumentation, and ultimately leading to modern fixation systems of segmental pedicle screw instrumentation ${ }^{17}$. The recent investigations to enhance arthrodesis of L5-S1 with various augmentation techniques have also contributed to improved outcome. However, determining where to stop the proximal fusion is still a controversial topic. Some surgeons prefer to go beyond the thoracic apex, up to $\mathrm{T} 2, \mathrm{~T} 3$ or $\mathrm{T} 4$, with greater operative morbidity ${ }^{18}$. Others end the fusion at the upper lumbar region increasing the risk of proximal junctional problems ${ }^{19,20}$. Review of the literature, however, shows a paucity of clinical studies evaluating the long-term outcome and complications associated with ending proximal fusions at different levels.
In surgical treatment of adult lumbar deformity with long fusions, two important anatomic characteristics of the thoracolumbar junction should be considered. Because of the transition from the immobile thoracic spine to the mobile lumbar spine, from the coronal facet plane of the thoracic vertebrae to the sagittal facet plane of the lumbar vertebrae, and from thoracic kyphosis to lumbar lordosis, the thoracolumbar junction is a mechanically weak area. Ribs tend to increase the stability of the thoracic spine by the thoracic cage per se. The thoracic cage effectively increases the transverse dimensions of the spine structure that increases the moment of inertia, resulting in added resistance to bending in the sagittal and frontal planes, as well as resistance to axial rotation. The upper ten thoracic vertebrae (T1 T10) have this mechanical support through the true ribs, but T11 and $\mathrm{T} 12$ have false ribs without costosternal articulation. If the whole lumbar vertebrae were fused, stress could concentrate at the thoracolumbar junction as a powerful bending moment and result in proximal failure. In the current study, surgical outcome was analyzed with attention to the extent of the proximal fusion. Proximal stability was reinforced by the chest cage in Group 3 with fusion beyond the thoracolumbar junction (i.e, up to T9 or T10). Group 2 with fusion up to T11 or T12 had costoverterbral articulation with floating ribs, and Group 1 with fusion up to L1 or L2 had no rib support. Significantly more proximal junction problems developed in Groups 1 and 2 than Group 3 with contrast to well-maintained coronal correction in all three groups.

Some authors have stated that maintenance and restoration of lordosis and sagittal balance in the fusion mass were more important than coronal correction in the surgical treatment of adult lumbar deformity ${ }^{21-23}$. However, in current study, proximal junctional problems developed in Groups 1 and 2 in spite of well-maintained lordosis of the fusion mass. Total lumbar lordosis and sagittal balance were deteriorated at most recent follow-up because of proximal junctional problems. Well-maintained sagittal alignment in Group 3 can justify the anatomical difference of Group 3 compared with Groups 1 and 2.

Swank et al. ${ }^{19}$ presented surgical outcomes in long lumbar fusions from L1 or L2 to S1 done for a variety of spinal pathologies including degenerative spinal stenosis, scoliosis, postlaminectomy syndrome, pseudoarthrosis and spondylolisthesis. Of the 20 patients, 7 patients required reoperations for fractures at or above the uppermost instrumented vertebra, adjacent segment degeneration or infec- 
tion. Only 2 patients had good or excellent clinical result. They concluded that instrumented lumbosacral fusions that begin at L1 or L2 have an unacceptably high mechanical failure rate in adult patients and cannot be recommended. In a recent presentation, Simmons et al. ${ }^{20}$ reported adjacent segmental problems in $60 \%$ of elderly patients who had lumbar fusions extending to $\mathrm{L} 1$ and in $56 \%$ of them to $\mathrm{L} 2$. Bridwell ${ }^{24}$ stated choosing the proximal fuion level requires identification of the stable, neutral and horizontal vertebra in the coronal and sagittal planes. He typically terminates the fusion proximally at T10 or T11 in his adult lumbar scoliosis patients.

The fusion level in Swank and Simmon's study was equivalent to our Group 1. In the current study, half the patients in both Groups 1 and 2 had proximal adjacent segmental problems in contrast to 1 out of 7 in Group 3. Deterioration of sagittal balance during follow-up was observed in 36\% of Groups 1 and 2, but none in Group 3. As mentioned above, if the proximal fusion is extended beyond the thoracolumbar junction up to $\mathrm{T} 9$ or $\mathrm{T} 10$, the bending moment between the instrumented and un-instrumented junction is decreased. The high success rate in Group 3 may be attributed to the unique anatomical characteristics of the thoracolumbar junction.

\section{Conclusions}

Proximal adjacent segmental problems and trunk imbalance occurred more frequently in proximal fusions that extended to the thoracolumbar junction (L2 T11) as apposed to the true rib area (T9 or T10) in surgical treatment of adult lumbar deformity. Fusion higher than T10 with restoration of sagittal balance is recommended in the surgical treatment of adult lumbar deformity.

\section{REFERENCES}

1. Farfan HF, Huberdeau RM, Dubow HI: Lumbar interververtebral disc degeneration: the influence of geometrical features on the pattern of disc degeneration--a post mortem study. J Bone Joint Surg Am 1972; 54A: 492-510.

2. Jackson RP, Simmons EH, Stripinis D: Coronal and sagittal plane spinal deformities correlating with back pain and pulmonary function in adult idiopathic scoliosis. Spine 1989; 14: 1391-1397.
3. Kostuik JP, Bentivoglio J: The incidence of low-back pain in adult scoliosis. Spine 1981; 6: 268-273.

4. Van Dam BE: Nonoperative treatment of adult scoliosis. Orthop Clin North Am 1988; 19: 347-351.

5. Dickson JH, Mirkovic S, Noble PC, Nalty T, Erwin WD: Results of operative treatment of idiopathic scoliosis in adults. J Bone Joint Surg Am 1995; 77: 513-523.

6. Balderston RA, Winter RB, Moe JH, Bradford DS, Lonstein JE: Fusion to the sacrum for nonparalytic scoliosis in the adult. Spine 1986; 11: 824-829.

7. Kostuik JP, Hall BB: Spine fusion to the sacrum in adults with scoliosis. Spine 1983; 8: 489-500.

8. Saer EH 3rd, Winter RB, Lonstein JE: Long scoliosis fusion to the sacrum in adults with nonparalytic scoliosis. An improved method. Spine 1990; 15: 650-653.

9. Emami A, Deviren V, Berven S, Smith JA, Hu SS, Bradford DS: Outcome and complications of long fusion to the sacrum in adult spine deformity: Luque-Galveston, combined iliac and sacral screws, and sacral fixation. Spine 2002; 27: 776-786.

10. Eck KR, Bridwell KH, Ungacta FF, et al: Complications and results of long adult deformity fusions down to L4, L5, and the sacrum. Spine 2001; 26: E182-192.

11. Tsuchiya K, Bridwell KH, Kuklo TR, Lenke LG, Baldus C: Minimum 5-year analysis of L5-S1 fusion using sacropelvic fixation (bilateral S1 and iliac screws) for spinal deformity. Spine 2006; 31: 303-308.

12. Perennou D, Marcelli C, Herisson C, Simon L: Adult lumbar scoliosis: epidemiologic aspects in a low-back pain population. Spine 1994; 19: 123-128.

13. Robin G, Span Y, Makin M, Steinberg R: Scoliosis in the elderly: idiopathic or osteoporotic. Proceedings of the 5th Zorab scoliosis symposium. London 1976.

14. Kim JH, Suk SI, Chung ER, et al: Epidemiologic study of lumbar scoliosis with plain abdominal X-ray. J Korean Spine Surg 2004; 11: 246-252.

15. Dick W, Widmer H: Degenerative lumbar scoliosis and spinal stenosis. Orthopade 1993; 22: 232-242.

16. Fellrath RF Jr, Hanley EN Jr: Causes and management of unstable degenerative spinal stenosis. J South Orthop Assoc 1996; 5: 221-228.

17. Cotrel Y, Dubousset J, Guillaumat M: New universal instrumentation in spinal surgery. Clin Orthop Relat Res 1988; 277: 10-23.

18. Cotler JM, Cotler HB: Spinal fusion: science and technique. New York: Springer-Verlag; 1990.

19. Swank S, Lonstein JE, Moe JH, Winter RB, Bradford 
DS: Surgical treatment of adult scoliosis. A review of two hundred and twenty-two cases. J Bone Joint Surg Am 1981; 63: 268-287.

20. Simmons ED, Huckell CB, Zheug Y: Proximal kyphosis, "Tapping Off Syndrmoe," and retrolisthesis secondary to multilevel lumbar fusion in the elderly patients. SRS annual meeting, Miami 2005.

21. Berven S, Deviren V, Demir-Deviren S, Hu SS, Bradford DS: Studies in the modified Scoliosis Research Society outcomes instrument in adults: validation, reliability, and discriminatory capacity. Spine 2003; 28: 2164-2169.

22. Booth KC, Bridwell KH, Lenke LG, Baldus CR, Blanke
KM: Complications and predictive factors for the successful treatment of flatback deformity (fixed sagittal imbalance). Spine 1999; 15: 1712-1720.

23. Farcy JP, Schwab FJ: Management of flat back and related kyphotic decompensation syndromes. Spine (Phila Pa 1976) 1997; 22: 2452-2457.

24. Bridwell KH: Selection of instrumentation and fusion levels for scoliosis: where to start and where to stop. Invited submission from the Joint Section Meeting on Disorders of the Spine and Peripheral Nerves, March 2004. J Neurosurg Spine 2004; 1: 1-8. 